\title{
Structural borrowing: The case of Kenyan Sign Language (KSL) and Kiswahili contact signing.
}

\author{
G. J. Jefwa
}

When two languages co-exist in the same environment, there is often some mixing of the systems. As can be expected, the dominant language will often have more effect on the minority language than vice versa. These generalizations apply to sign language situation. Sign languages which co-exist with spoken languages often borrow characteristics of the spoken languages in varying degrees. Pakhurst and Parkhust (1995:8)

\begin{abstract}
Kenyan Sign Language (KSL) is a visual gestural language used by members of the deaf community in Kenya. Kiswahili on the other hand is a Bantu language that is used as the national language of Kenya. The two are world's apart, one being a spoken language and the other a signed language and thus their "... basic structural units are of necessity fundamentally very different..." (Valli and Lucas: 1996). However, preliminary investigations have revealed that sometimes Kenyan signers use KSL signs while mouthing in Kiswahili. However, this does not necessarily put a case for the existence of a Kiswahili sign language since KSL is a natural language with its own autonomous grammar distinct from that of any spoken language.

In this paper, we shall argue that the Kiswahili mouthed KSL signs are an outcome of contact between KSL - Kiswahili bilinguals and their hearing Kiswahili bilingual counterparts. Following Valli and Lucas (1996). We will further argue that there are no contact signs in a situation where a sign language is in contact with a spoken language this being due to the fact that spoken languages do not have signed forms and thus no new signs are formed due to this contact. The signing in a KSL - Kiswahili contact results into a phenomenon called contact signing. The paper further argues that as a result of interaction between KSL and Kiswahili, KSL users sometimes code switch/code mix in a contact situation that involves hearing Kiswahili users and surprisingly in some deaf - deaf contact situations. This interaction has led to what Davis (in Valli and Lucas op cit) call "unique phenomenon" that manifests itself in mouthing, finger spelling, code switching/mixing and contact signing.
\end{abstract}




\section{Introduction:}

\subsection{The Kenyan Language situation.}

Kenya has over 46 distinct languages, which are linguistically classified into the following three main language families:
(i) Bantu
(ii) Nilotic
(iii) Cushetic

The Bantu languages are divided into fifteen (15) main languages. The Nilotic languages are divided into Dholuo language and para-nilotic languages which comprise four sub languages. The Cushetic languages on the other hand are also composed of four sub languages. Apart from this linguistic diversity resulting from these native languages, the Kenyan linguistic scene also records the existence of several "foreign" languages including: English, French, Japanese, Hindustan and Arabic which can be seen as Kenya Languages in their own right. The offshoot of the above scenario is the highly multilingual nature of the Kenyan society. From the above we can conclude that Kenyans have a repertoire of codes available for their everyday use.

Most Kenyans speak up to three languages namely: first language, Kiswahili (national language) and English (official language). According to Okombo (1994), the current language situation in Kenya, as in most of Africa, is that some kind of diglossia already exists, involving the selective use of an ethnic language (Kiswahili/ first language) in one set of circumstances and the use of one official often foreign language ( English) in another set. Ironically, the classification of the different languages found in Kenya done above leaves out Kenyan sign language (KSL). This is understandable since KSL can be viewed as a marked form of communication in a situation where all the languages mentioned above are spoken languages. KSL is marked because it is visual based unlike the spoken languages that are sound based. Spoken languages and signed languages use different modalities for communication. That is, "the basic structural units are of necessity fundamentally very different: morphemes composed of sounds are articulated in specific manners and places in the vocal tract, while morphemes composed of parts are articulated by the hands face and body.

However, since the deaf do not live in isolation and since they form a co-culture in the dominant culture of the hearing, the members of the deaf community interact with members of the hearing community as such this linguistic interaction results into Language contact between KSL and different spoken languages notably English and Kiswahili. Some of the effects of the contact between English 
and KSL have been documented (cf. Omangi 1999). The focus of this paper however, is on the effects of the contact between Kiswahili and KSL.

\subsubsection{The Kenyan Deaf and Kenyan Sign Language}

Contact arises when two cultures meet. Culture being the values, beliefs, perceptions and behaviours shared by members of a community. Culture though is primarily learned through language. The Kenyan deaf people constitute a culture in the sense that they share the same values, beliefs, perceptions and behaviours. One of the behaviours that they share is the same language. The language they share is called Kenyan sign Language and which as has been pointed out earlier is fundamentally different from spoken language.

Since deafness results from non-functionality of one's auditory channels, deaf people cannot make use of the vocal - auditory channel for communication. This puts the deaf in a unique position since speech (the audio oriented mode used by the majority) is unnatural to them, and thus they turn to a visual mode of communication - sign language. For the Kenyan deaf their language is Kenyan Sign language (KSL). KSL can be defined as a patterned visual gestural system used by deaf Kenyans (and hearing Kenyans who learnt it) for communication. Sign language uses signs that are culturally determined. "Every sign language evolves as a response by a given community of deaf people to the stimulation its members get from their immediate environment. What all the deaf people of the world share is the natural ability (i.e. their innate capacity) to learn a language and to contribute to the continuous enrichment of whatever language they use" (Okombo 1994). In this case then every deaf person uses the language of his / her environment an environment that has a national catchment area. However, it is important to note that unlike spoken language, sign language "... contains an unknown number of national, regional and cultural varieties." Bergman (1994). These varieties are usually aggregated at the national level, ignoring the extremely localized peculiarities. Thus we can talk of national sign languages such as Kenyan Sign Language (KSL) Tanzanian sign Language (TSL) British sign Language (BSL) etc. As to how a common sign Language emerges in the highly ethnically heterogeneous community of deaf Kenyans, see Okombo and Akach (1997). Members of the deaf culture therefore are users of a different language from that of the dominant hearing culture. This makes sense when we think of language as a system that consists of a series of conventional and arbitrary symbols (audio or visual) determined by a culture. With the hearing using an audio-based symbol system and the deaf using a visual based one.

According to Woodward (1972), the term deaf culture refers to two groups. There is the deaf with a small' $d$ ' and deaf with a capital ' $D$ '. Deaf with a small' $d$ ' refers primarily to hearing loss. Deaf with a capital " $\mathrm{D}$ " on the other hand refers to social collectivities. Parkhust and Parkhust (1995:7) People who are deaf may or may not identify with the deaf community. Deaf with a capital ' $D$ 'refers to those people who identify themselves as members of the Deaf culture who need not necessarily be 
deaf. The members of the Kenyan deaf community who are users of Kenyan sign language do not live in isolation within the Kenyan society. They interact with their hearing counterparts in church, hospitals, police stations etc. Thus the deaf and the hearing are in constant contact and thus their languages are also in contact.

\section{Language contact}

\subsection{KSL vs. Kiswahili}

When two or more languages come into contact bilingual or multilingual communication takes place. Most deaf Kenyans are fluent in KSL. Some have some level of fluency in English. The little English they learn and use is a direct result of their schooling experiences where "the traditional insistence in deaf education setting in the use of the spoken language of the community or the use of some combined form of signing and speaking, with exclusion of sign language. ..." is the order of the day. This scenario is the direct result of contact between a sign language and a hearing language in this case English. English is an official language in Kenya and the medium of instruction in schools ironically including schools for the deaf. Kiswahili on the other hand is the national language of Kenya. However, most deaf people don't come into contact with Kiswahili since it is not taught in most of their schools. A few Deaf schools which have been noted to teach Kiswahili as a subject include: Nyangoma Technical School for the Deaf, Ziwani School for the Deaf, Machakos School for the Deaf, Rev. Muhoro School for the deaf and Kerugoya School for the deaf. In these schools however, the teaching of Kiswahili is done haphazardly thus the teaching is not at par with the hearing schools. In deaf schools, the teacher reads and writes on the board and in turn instructs the students to write. The students write Kiswahili words without understanding their meanings. This is exemplified from a personal anectode from Washington Akaranga (a deaf adult KSL instructor):

When I went to Machakos school for the deaf the students told me they knew Kiswahili and proceeded to write many words on the board, I was surprised since as a deaf adult myself I only knew very few Kiswahili words. I asked them to tell me what those words meant and none had an idea.

The personal testimony above gives an overview of the situation in most deaf schools. Our preliminary investigations also revealed that in the schools where Kiswahili is taught, its teaching begins from class six and not at any lower classes. This also sheds some light on the attitude teacher and educators involved in deaf education have towards Kiswahili. The majority of deaf schools don't even attempt to teach the language since they have no idea how to do it to deaf children. From the above, we can conclude that the little Kiswahili some deaf people know comes mainly from learning from family members or from other deaf people especially those who went deaf after a hearing period. Similarly, "though code switching bilinguals may be highly proficient in both languages, balanced proficiency is by no means a prerequisite. Indeed code switching is possible with a limited knowledge" (Aver 1998). 
This is the case in the KSL-Kiswahili contact situation where despite the limited knowledge the deaf have of Kiswahili they still are able to code switch to Kiswahili by mouthing Kiswahili words with KSL signing.

It is important to note that there can be many different types of participants in a contact situation as long as the deaf are concerned:

- Deaf participants vs. hearing individuals.

- $\quad$ KSL English bilinguals.

- $\quad$ Deaf participants who were mainstreamed at an early age and learnt to sign late.

- Hearing children of deaf parents who can be KSL - English bilingual who learnt KSL at home natively.

- Hearing individuals who know SL

- Hearing individuals who are English monolinguals

The possible language contact situation involving the deaf includes:

- Deaf bilinguals with hearing bilinguals

- Deaf bilinguals with deaf bilinguals

- $\quad$ Deaf bilinguals with hearing spoken English monolingual

(Valli and Lucas 1996)

\subsection{The situation of the Deaf in Kenya}

Most deaf Kenyans are fluent in KSL and are marginally fluent in English or Kiswahili. These are the ones we will refer to as deaf bilinguals. Deaf bilinguals are those deaf people who have knowledge of KSL learnt as a first language from their parents or from their peers at an early age while in boarding school. Okombo and Akach (1997) under score the importance of schools when they assert: "the single most important environmental stimulus for the development (emergence and growth) of KSL in Kenya has been the formation of communities of deaf persons in schools and deaf institutions." The deaf bilinguals also have a degree of exposure to English or Kiswahili. This exposure could be from school via speech training, through media, through lip-reading etc for English and from family members, teachers (privately), from other deaf (especially gone deaf people) for Kiswahili.

Hearing bilinguals on the other hand will be speakers of Kiswahili who learnt KSL as adults through interaction with the deaf or through formal instruction. Thus the 
participants in the KSL - Kiswahili contact situation make use of both the vocal and visual channel. Vocal channel mainly used by the hearing speakers of Kiswahili and the visual channel, which also includes the use of both manual and non-manual signals for the deaf bilingual. It is important to note that both channels are also available for both. The participants in this kind of contact situation have hands, mouth and face available for including of linguistic messages" (Valli and Lucas 1996).

The above distinction between deaf bilinguals and hearing bilinguals is important since it brings to the fore very important issues regarding this type of contact situation. When a deaf person is in a communication situation with another deaf person their signing is different as compared to when they are signing with a hearing individual. Similarly, deaf people can start signing to a hearing individual and end up switching to mouthing in another language e.g. English or Kiswahili if the interlocutor's ability to hear and use the language is known. For example, when a deaf person is signing to a hearing person before they realize that the person is hearing, they may not use mouthing or switch or mix codes. However, the moment they realize that the interlocutor is hearing, they may mouth some words or even code switch or code mix through finger spelling etc. What then happens, when bilinguals come into contact?

\subsubsection{Possible outcomes of KSL bilinguals and Kiswahili bilingual contact.}

Interaction between two languages results in some characteristics of each of these languages influencing each other. However, "the dominant language will often have more effect on the minority language than vice versa" (Parkhurst and Parkhust 1955:8). In the case of the KSL deaf bilingual vs. Kiswahili bilinguals contact situation the dominant language is Kiswahili, which is the national language of Kenya. This contact has several outcomes, which we shall outline below:

- Borrowing

- Code switching/code mixing

- Contact signing

- Unique phenomena: - Finger spelling

- Mouthing

\section{Borrowing}

Linguistic systems are normally affected by language contact. If the effect results in the borrowing of the phonological, morphological or syntactic features we talk of structural borrowing which is the concern of this paper. According to Crystal 
(1980:27) borrowing refers to linguistics forms being taken over by one language or dialect from another.

Borrowing is a phenomenon that every language goes through. In other words there is no pure language. However in the case of SL in general and KSL in particular there is a general tendency to question the authenticity of KSL as an indigenous language on the grounds that it has signs from other sign languages. This of course is a product of contact between KSL and other sign languages, which is a natural occurrence in contact situations. (This however is not the concern of this paper). Borrowing is one of the ways in which all natural languages acquire new items as part of what is called corpus development i.e. the growth of the body of the language itself, its vocabulary, its grammatical structure and the meaning of the items in it, (Okombo 1994:49).

According to Valli and Lucas (1996), it is impossible for any sign language to borrow from a spoken language though borrowing is possible between one sign language and another. This is because spoken languages do not have signed forms and sign language cannot borrow spoken phonology, they argue. However, from our investigation of KSL - Kiswahili contact we have found evidence of borrowing which we will discuss.

One thing for sure is that the intensity of contact affects linguistic borrowing. The KSL- Kiswahili contact is characterized by low intensity contact and thus only limited instances of borrowing can be observed. This contact we believe, inter alia, leads to code switching. However the line that distinguishes borrowing and code switching is sometimes seen as being very narrow. Those who hold this view use criteria such as the degree of phonological, morphological and syntactic integration undergone by the item in question. If it is fully integrated, the element can be regarded as borrowing. The Kiswahili lexical items discussed below are examples of borrowing since they are fully integrated into KSL. This is illustrated by the fact that they are not an exclusivity of deaf- hearing contact situation but also of deaf- deaf contact situation. In other words these Kiswahili lexical items are used in the two situations. The use of these lexical items among KSL deaf users is significant in showing how integrated they are.

The contact between KSL and Kiswahili has brought about some Kiswahili words finding their way into KSL. However we should note that these words do not enter Kiswahili as words on their own per se but they are used with existing signs thus no new signs are formed to accommodate them. The existence and use of these words in Kiswahili, we will argue, is a case of borrowing, as an outcome of contact and we shall also argue that it leads to "contact signing." a phenomena we shall deal with shortly. Some of the Kiswahili words that are used in KSL include:

$\begin{array}{ll}\text { MZUNGU } & \text { (A European) } \\ \text { JOGOO } & \text { (A cockerel) }\end{array}$

BASI (That's all)




$\begin{array}{ll}\text { BADO } & \text { (Not yet) } \\ \text { SAFARI } & \text { (Journey) } \\ \text { MIA } & \text { (One hundred) } \\ \text { HAPA HAPA } & \text { (Just here) } \\ \text { POA } & \text { (Fine) }\end{array}$

WEE! (An exclamation for YOU!)

The following are examples of Kiswahili lexical items that have been borrowed into KSL as a result of the contact between KSL and Kiswahili as used in KSL sentences. For example:

1. ME I DON'T KNOW/ME DON'T KNOW /ME TEACH DEAF

I don't know. I don't know. I teach the deaf people speech using ORALISM BASI / FINISH/

The oral method. BASI. That's all.

2. NOW TEACHER START SHARING BOTH/START BOTH

Now the teacher started using both. They started sharing both SHARING BEFORE NOTHING/ NOW USE BOTH/ SOMETIMES before then they were using neither. They sometimes went to teach GO SCHOOLS TEACH SL/ DEAF COMMUNICATION/ THIS in Deaf schools using KSL. These were arrangements with some TOGETHER DEAF SCHOOL BUT OFFICIAL WITH

deaf schools Since such arrangements with the government were nonGOVERNMENT BADO

existent.

3. OTHER BORROW ASL COME KENYA/ THEMSELVES TELL

Some Kenyans went to America and brought borrowed ASL and DEAF/ TELL DEAF YOU FATHER WHERE? FATHER YOURS they asked Kenyan deaf people where is your father? Where is your WHERE/ DEAF KENYA TELL /ME UNDERSTAND BADO / MY Father The deaf Kenya said I don't understand my Jogoo is at home. JOGOO HOME THERE/

4. HAPPEN ONE MZUNGU COME KENYA/ THIS MZUNGU

It happened one European came to Kenya. This European was deaf. HIMSELF DEAF/THIS MZUNGU HIMSELF COME SAFARI He had come on safari but found a deaf a deaf Kenyan signing ONLY FOUND DEAF PERSON THERE. SIGNING / BASI in example 1 above is signed with open palms facing outwards towards the listener starting at the center and then moving in different directions. 
BADO in example 2 above on the other hand, is signed with the protruding thumb hand form in front of the signer moving from side to side.

In example 3 above, Two Kiswahili words are used, BADO appearing for the second time and JOGOO is signed with open palm hand forms with fingers wide apart one on the forehead (thumb touching the fore head) and the other on the chin (thumb touching the chin) the one on the fore head is oriented upwards while the one on the chin is oriented downwards.

In example 4 above MZUNGU, is signed with one open palm facing upwards and the index finger moving up and down on the open palm. It is important to note that in this example the word MZUNGU appears three times. Repeated cases of code switching are conversational strategies used to add emphasis to certain components.

The other signs identified in this paper are MIA, SAFARI, HAPA, POA and WEE!

SAFARI is signed with two index finger hand forms in front of the signer on the sides moving out wards to and fro. MIA on the other hand is signed with the index finger hand form on the lips with one movement in front of the signer. HAPA is signed with two open palm hand forms on the sides of the signer with movement up and down. POA on its part is said to be used mainly by Tanzanian deaf people but is slowly finding its way into KSL (an example of borrowing from one SL to another). It is signed with two open palm hand forms bent at the knuckles facing the addressee moving back and forth in front of the signer. The slowness of the movement indicates the coolness of the situation. POA can also be signed with two thumbs up hand forms starting in front of the signer and then moving side ways. Again in this sign, the slowness of the movement indicates the coolness of the situation.

WEE! Is an exclamation. It is mostly used by deaf women for example when talking about childbirth they would state" its painful WEE!" It is signed with index finger hand form pointing at listener emphatically. One point worth noting is that all these Kiswahili words are mouthed in Kiswahili while accompanying KSL signs. In other words, the basic grammar in use belongs to KSL and the switches noted are lexical substitutions from another code - Kiswahili.

As pointed out earlier these Kiswahili words as used in KSL are only mouthed in Kiswahili but they are not signs. The signs used with them are basically KSL sign with KSL meaning and function. Let us now turn to one of the outcomes of these lexical items entering Kiswahili- the phenomenon of mouthing.

\section{Mouthing}

These lexical items when used in KSL, take the mouth pattern of Kiswahili but the form and function of KSL signs. Woll (1990:958) asserts that signers use mouth patterns as a result of their exposure to spoken language. Mouthing forms the "spoken" components of Sign language. KSL users tend to mouth certain signs t match with words in a spoken language in this case Kiswahili. The Kiswahili words found in the examples above are normally mouthed while signing their KSL 
equivalents. That is, they are produced with KSL signing accompanied by mouthing. Davis in Valli and Lucas (1995:81) identify different types of mouthing or mouth patterns.

Full mouthing: Here words are pronounced without voice.

Reduce mouthing: the words are not fully pronounced.

Lexicalised mouthing: E.g. the mouth configuration of FINISH and HAVE (ASL) which clearly derive from English pronunciation but have become part of ASL signs.

The above Kiswahili words as used in KSL fall under the third category. They take the mouth configuration of the Kiswahili words and thus it is clear that they derive from Kiswahili. However, over a long period of use, they have become part of KSL. The fact that they have become part of KSL can be proved since they are used not only in deaf -hearing contact situation but also with an exclusively all-deaf audience. However it is important to note as Mesthrie et al (2000:249) noted that the borrowing of a word does not presuppose knowledge of the language from which it is taken. Apart from those deaf people who became deaf after acquiring Kiswahili the rest of the deaf of the deaf have no knowledge of the Kiswahili language.

Mouthing or what Spence and Woll (2005) call the spoken components of SL have various uses:

a) to represent spoken language mouth pattern in combination with signs

b) to represent spoken language mouth patterns with first letter signs, and ;

c) to distinguish other manual homonyms

The Kiswahili mouthing discussed above is mainly used to represent spoken language mouth pattern in combination with signs.

\section{Code switching/code mixing}

Aver (1998) views code switching and code mixing as the same. He asserts that they occur when two languages are juxtaposed in discourse and or within a sentence variously called language alternation, code switching or code mixing.

When the Kiswahili words mentioned above are used in a deaf KSL bilingual vs. hearing Kiswahili bilingual situation, we can say they are used for the purposes of establishing rapport and also establishing social identity in as far as the KSL user is aware that the hearing bilingual understands Kiswahili. In this way the use of these Kiswahili words can also be seen as code switching. Where a deaf person who also knows Kiswahili can use KSL and then momentarily switch to mouthing in the KSL signs in Kiswahili. In this case items produced are KSL lexical items with KSL meaning and function. They do not have Kiswahili forms and meaning. The use of Kiswahili words as exemplified above resulting into contact signing can also be viewed as code switching or code mixing. In this paper, following Aver (Op cit) we shall use the two terms interchangeably wherever applicable since there is switching from one code to another which leads to mixing of codes which in turn gives us KSL 
forms with Kiswahili mouthing. Bokamba (1989) defines code switching and code mixing as follows:

Code switching: "An inter sentential event, the embedding or mixing of words, phrases and sentences from two codes within the same speech event and across sentences boundaries."

Code switching can manifest it self in four different ways:

(i) Tag switching: Here tags and certain set phrases in one language are inserted in an utterance of another.

(ii) Intra sentential switching: Here, switching occurs within clause or sentence boundaries.

(iii) Inter sentential switching: Here switching occurs at clause or sentence boundary where each clause or sentence is in one language or the other. For example starting a sentence in one language and finishing it in another.

(iv) Intra word switching: Here change occurs within a word boundary. For example, KUENJOY which is composed of an English word enjoy with a Kiswahili prefix KU- meaning 'to'

Code Mixing: An intra sentential event, the embedding or mixing of various linguistic units such as affixes, words, phrases, and clauses from two distinct grammatical systems or subsystems within the same sentence and same speech situation.

Though the two are at times viewed differently our position is that the two can be used interchangeably. That is wherever there is code switching from one language to another code mixing is inevitable. The switch witnessed in the KSL sentences above fall under inter- sentential switching where the speaker starts in one language and ends up in another e.g. in example 1 and 2 above. Some of the examples can be classified as examples of Tag switching where tags and certain set phrases from one language are inserted into an utterance in another language for instance examples 3 and 4 above.

Mesthrie et al (2000:437) define code switching as including alternation in channel (from oral to manual) as well as switching across the sign continuum (from SL to manual sign codes). He also adds that this switching is a pervasive feature of deafhearing and deaf-deaf communication. Thus both code switching and code mixing refer to speech that draws from at least two languages combined in different ways. When an individual who is bilingual alternates between two languages during his or her speech with another bilingual individual this is said to be code switching or code mixing.

Code mixing and code switching often serve important functions mainly within ethnic and group boundaries. Among minorities e.g. KSL users, the 'we' code is 
used to signify in-group, informal and personalized activities. In the other language the 'they' code is used to mark the out-group, more formal and distinct events. The changing of language by speakers indicates their attitudes of what is being said. The prestige of knowing the out group language (in this case Kiswahili) also contributes to the reasons for switching or mixing. For the deaf KSL users adding Kiswahili words gives them the prestige of knowing the out-group or dominant language. When this mixing or switching occurs with fellow deaf people the speaker is identifying him/herself with the dominant language or is trying to show his or her expertise or knowledgability of the language. If it occurs with hearing people the speaker uses it to socially identify him/herself with the hearing.

We can argue that the mixing of codes in many ways corresponds to the deaf elites 'unmarked' way of speaking for which using Kiswahili words is a way of identifying with the hearing. This is true even when the deaf use Kiswahili mouthed words to accompany KSL signs in deaf-deaf contact situation since then they are able to prove their knowledgeability of the language to other deaf people. The issue of prestige also comes in here. In the KSL -Kiswahili contact situation, the two languages are not of equal prestige with Kiswahili being more powerful / prestigious. The two are said to be in a super or substral relation and not in an adstratal relation, which they would have been if they were of equal prestige.

\section{Finger spelling}

Code mixing manifests itself in Kiswahili through the use of finger spelling, or manually coded alphabet. KSL uses the one handed international manual coded alphabet. This aspect of contact signing is a direct result of the role of the educational system in which spoken languages are used and thus deaf people can use finger spelling to spell words or objects that do not have signs or those whose signs they have forgotten when in contact with hearing bilinguals. Finger spelling according to Davis in Valli and Lucas falls under unique phenomena. Finger spelling allows the representation of the orthographic forms of the letters of the alphabet manually. This is a unique borrowing between the phonology of one language - KSL and the orthography of a spoken language English or Kiswahili and it is as a result of contact.

Deaf Kiswahili bilinguals can at times spell Kiswahili words using their fingers. This allows deaf people to code mix i.e. use Kiswahili words in KSL sentences in the process code mix Kiswahili words with KSL signing. This type of code mixing is only possible for deaf people who know how to read and write a certain spoken language in this case Kiswahili. Contact can also result in different changes if its effects result in the borrowing of the phonological, morphological or syntactic features we talk of structural borrowing. Structural changes can be at the morphological and syntactical levels; these changes may manifest themselves in the order of signs.

Morphology deals with how a language uses smaller units to get to larger units it is thus concerned with word or sign formation. Lexical borrowing which is what the Kiswahili words borrowed into KSL is all about fall under morphology. Syntactically, the signs may follow the pattern of the second language. In this case it may follow the Kiswahili sentence pattern (word order). However, due to the low intensity of 
contact between KSL and Kiswahili there are no changes noted at the sentence level as a result of that contact.

As Aver (1998) puts it, "since code switching serves to conceptualize certain language activities the utterance units affected by the switch must be large enough to constitute such an activity. For this reason code switching does not provide interesting data for syntactic research." The unique nature of contact signing however allows KSL to combine KSL lexical items in a reduced Kiswahili syntactic system.

\section{Contact signing}

As noted above, the KSL - Kiswahili contact situation which is a linguistic contact situation leads to 'contact signing'. According to Fischer (1998.314) “Contact signing is characterized as an interface between deaf signers and hearing speakers". Contact signing is thus a product of interaction. However one interesting thing is that contact signing does not necessarily occur in the presence of hearing people in other words it is not an exclusivity of KSL bilinguals and Kiswahili bilinguals, it also occurs in exclusively deaf - deaf contact situation.

The outcome of contact that stems from those discussed above i.e., mouthing, code mixing/switching and finger spelling is contact signing. Contact signing in this case is the result of the contact between KSL and Kiswahili. The signing that comes out of this contact situation has features of both. The mouthed lexical items borrowed from Kiswahili after a time of use become part of KSL and thus contact signing then is not only used with hearing people but also between deaf and deaf.

Another interesting phenomenon noted by Lucas is "following spoken language criteria literally"- where someone literally stops signing and starts speaking or vice versa, e.g. "hearing KSL bilinguals may produce KSL sentences or signs with each other (with no deaf people present) in the course of English or Kiswahili conversation."

This is also true in Kiswahili where two hearing KSL bilinguals may in the course of conversing in Kiswahili stop and use KSL. Also two deaf Kiswahili bilinguals may in the course of conversation in KSL switch to spoken language especially if they became deaf after acquisition of a spoken language. Similarly, in a deaf- hearing contact situation, a deaf person may switch to mouthing in Kiswahili when he becomes aware that the hearing person is conversant with the language.

\section{Summary}

In this paper, we have tried to show how contact between KSL and Kiswahili, two languages whose basic structural units are fundamentally very different have resulted in some form of borrowing, with some Kiswahili mouthed words being used together with KSL signing. We have pointed out that this happens despite the limited knowledge KSL users have of Kiswahili, which has a low intensity contact with KSL. The borrowing of Kiswahili mouthed words into KSL we have 
argued is structural borrowing, which involves borrowing of phonological (finger spelling) in morphological (lexical) and syntactic (word order) features. The contact between the two languages leads to different outcomes (apart from borrowing) which we have outlined as: code switching/ code mixing, and what Valli and Lucas, call unique phenomena that is finger spelling, and mouthing. We also argue following Valli and Lucas that a KSL signing situation that makes use of Kiswahili mouthed words is basically contact signing. In this situation the signs produced have KSL form and function - the basic grammar remains KSL and the switch can be viewed as lexical substitutions from another code- Kiswahili through the phenomena of mouthing.

References

Akach, Philemon, O (1991) Sentence types in Kenyan Sign Language: A structural approach. An unpublished M..A. Thesis. University of Nairobi.

Aver, Peter (1998). Towards a dynamic typology of bilingual speech. In Mesthrie, Rajend et al. (2000) Introducing Sociolinguistics. Edinburgh University Press: Edinburgh.

Bergman, B. (1994). Studies in Swedish Sign Language, Stockholm, University of Stockholm,

Crystal, D. (1980) A Dictionary of Linguistics and Phonetics. Oxford: Blackwell.

Bokamba, E.G. (1989). Are there syntactic constraints on code-mixing? London: Pergarnon Press.

Fischer, S. (1998). Critical Periods for Language acquisition: Consequences for Deaf education. In weasel, A(ed) Issues Unresolved New Perspective on Language and Deaf Education, 9-26. Washington D.C. Gallaudet University Press. Mesthrie, R. et al. (2000). Introduction to Sociolinguistics. Edinburgh University Press Muysken, Pieter (2000) Bilingual speech: A typology of code switching. Cambridge, Cambridge University Press

Okombo, Okoth (1994). Kenyan Sign Language: Some attitudinal and Cognitive issues. In the evolution of a language community. In Bilingualism in Deaf Education in I. Alilgren and K. Hyltenstam (ed): 37-54 Hamburg: signum

Okombo, Okoth and Akach Philemon (1997) "Language convergence and phenomena in growth of a national sign language in Kenya." In international journal of sociology of language: sociolinguistic issues in sub-Sahara Africa. Vol. II 125 Aldulaziz, M H (ed) moutonde Gruyter: Berlin

Okombo, O., Akaranga, W., Mweri, G.J. and Ogutu, A.T.A. (2006). Introduction to theory and skills of teaching Kenyan Sign Language: A handbook for teachers. Nairobi: Kenya society for Deaf children.

Omangi, D. N.C. (1999) Lexical borrowing in Kenyan Sign Language

(KSL) An unpublished M.A. thesis, Moi University.

Parkhurst, Stephen J. and Dianne Parkhurst. (1995). "Language and the Deaf."Notes on Linguistics 71: 7-15.

Spencer, S.R. \& Wool, B. (2005). The linguistics of British Sign Language: an introduction. London: Cambridge University Press.

Valli, C, and Lucas, C. (1996). Linguistics of American Sign Language: An introduction. Gallaudet University Press: New York.

Woodward M, and Inglehart, R.F. (1972) “Language conflicts and political community", in: Language and social context, Giglioli, P., ed., New York, 358-377.

Wool, B. (1990). 'International perspective on Sign Language Communication', International journal of Signed Linguistics 1, 107-12 